\title{
ROLE OF A FIELD HOSPITAL IN COVID-19 PANDEMIC
}

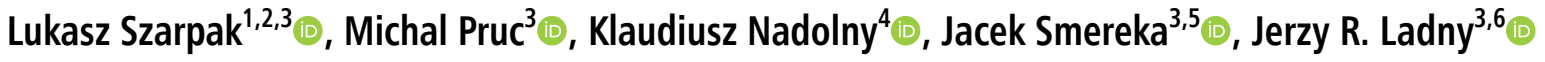 \\ ${ }^{1}$ Maria Sklodowska-Curie Bialystok Oncology Centre, Bialystok, Poland \\ ${ }^{2}$ Maria Sklodowska-Curie Medical Academy in Warsaw, Poland \\ ${ }^{3}$ Polish Society of Disaster Medicine, Warsaw, Poland \\ ${ }^{4}$ Department of Emergency Medical Service, Higher School of Strategic Planning in Dabrowa Gornicza, Poland \\ ${ }^{5}$ Department of Emergency Medical Service, Wroclaw Medical University, Wroclaw, Poland \\ ${ }^{6}$ Clinic of Emergency Medicine and Disaster, Medical University Bialystok, Poland
}

KEY WORDS: SARS-CoV-2, COVID-19, pandemic, field hospital, mobile hospital, medical treatment, organization

Disaster Emerg Med J 2020; 5(4)

At the end of 2019, a new type of coronavirus appeared in China - SARS-CoV-2, which challenged healthcare facilities around the world. Currently, according to Johns Hopkins University, there are more than 54.615.099 confirmed cases of COVID-19 infection worldwide. In many regions, the demand for in-hospital emergency health services has exceeded the capabilities of hospital systems, and it was necessary to create field hospitals used as part of a response strategy $[1,2]$. This strategy involves relieving the burden on specialist health care facilities by transporting patients in mild to moderate conditions who, for many reasons, have to be taken care of in the hospital to field hospitals. Many patients in mild to moderate conditions use beds in specialist centres without requiring constant specialist supervision. Transportation of such patients to field hospitals will provide free space for patients in critical conditions and will allow to provide them with appropriate care, which in many cases would be impossible due to lack of free hospital beds. As the examples from China, which after the experience with SARS were prepared for a possible new pandemic on a large scale, began to create field hospitals, numerous medical staff from all over China were sent to mobile hospitals in the most vulnerable areas. Two hospitals were established for patients in critical conditions and fifteen for patients with mild symptoms. During the epidemic, mobile field hospitals played a great role in fighting the epidemic. This strategy has yielded very good results and allowed both to relieve the burden on specialist facilities and to limit the spread of the virus [3]. In the early weeks of the COVID-19 pandemic, a large number of patients with acute respiratory failure represented a very serious burden on American health care systems. In Massachusetts, predictive models showed that up to 15.000 hospital beds were needed to handle the sudden increase in the number of cases, while the Massachusetts Department of Public Health reported that there were only 11.000 before the pandemic. These models showed that at peak incidence during the first wave of infections, COVID-19 beds might run out in the first weeks of April [4]. It became necessary to establish field hospitals, which resulted in the first field hospital in Massachusetts in the Worcester congress centre in early April. Many other states have taken similar steps and started field hospitals, such as those in New York and Boston. More than 41.000 people are currently being hospitalized because of COVID-19 in the United States, a 40 per cent increase over the past month according to the COVID Tracking Project in October, which further highlights the need to build and use field hospitals to prepare for the huge number of patients. Nowadays, apart from China and the United States of America, similar solutions have already been used practically all over the world, both in the form of temporary expansion of the hospital base and huge facilities such as congress halls or stadiums transformed into field hospitals $[5,6]$. An example of such a solution is the Temporary Hospital 


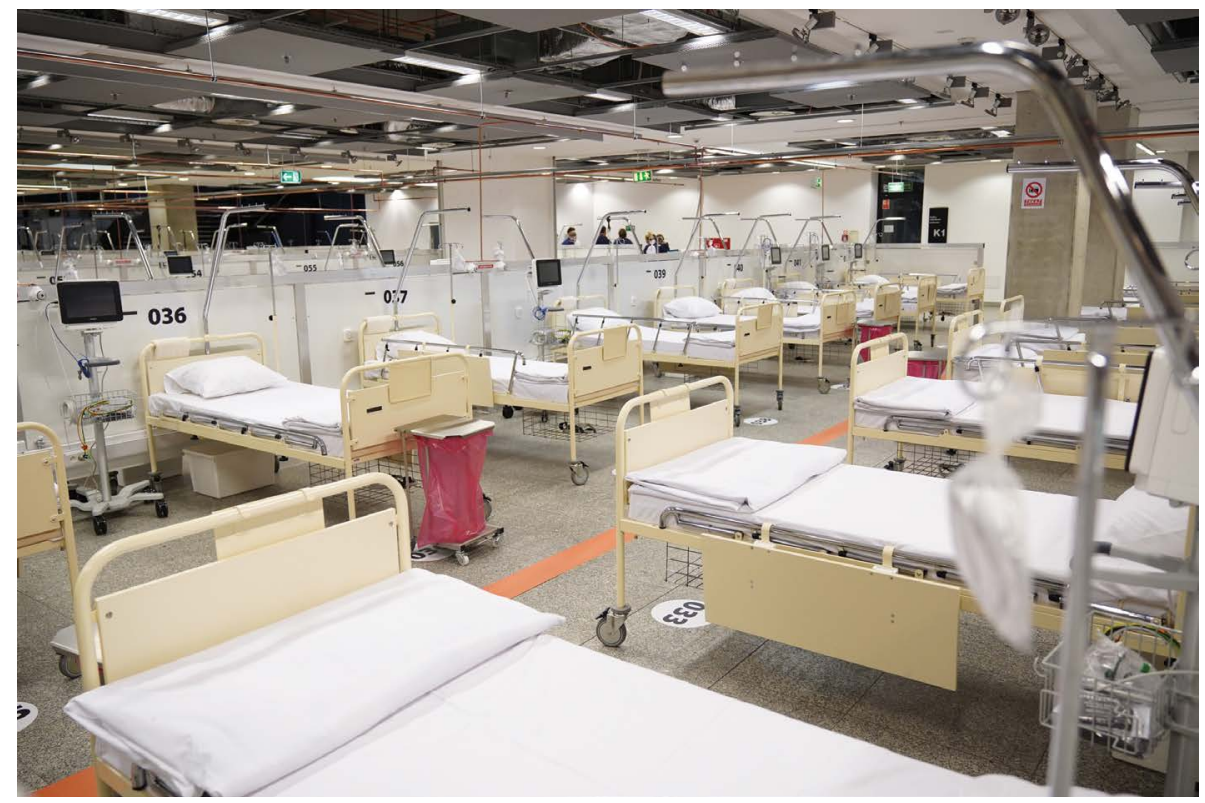

FIGURE 1. An example of the arrangement of conservative beds in a temporary hospital

of the Central Clinical Hospital of the Ministry of the Interior and Administration in Warsaw created in one of the most recognizable buildings in Warsaw - the National Stadium. As of 20 November 2020, the hospital has 500 beds dedicated to patients with COVID-19, with the possibility to extend the number of beds to 1200 .

The current criteria for admission to a Temporary Hospital may raise doubts both among medical personnel and the public, but this is due to a misunderstanding of the concept of creating temporary hospitals and their specificity. As already mentioned in the introduction, temporary hospitals do not fulfil the function of specialized hospitals and their role is limited to relieving specialized inpatient hospitals of patients in mild and middle status, to unblock hospital beds for patients requiring specialized treatment, including, among others, interventional cardiology, neurology, oncology or surgery. For example, Wang et al. indicated that patients were only admitted to a Mobile Cabin Hospital if they met all of the following criteria: 1. mild or moderate symptoms; 2 . normal activities of daily living; 3 . no important organ dysfunction; 4. no psychiatric history; and 5. resting pulse oxygen saturation $>95 \%$ [7]. In turn, $\mathrm{Xu}$ et al. indicated that in the event of a patient's condition deterioration they were quickly transferred to the designated higher-level hospitals. The inclusion criteria involved: 1 . met the criterion of severe or critical; 2. lung imaging showing a greater than $50 \%$ progression of lesions within 24-48 h; 3. development of a basic disease, such as hypertension, diabetes, coronary artery disease, etc. the patient should be transferred to the designated hospital [8].

It is extremely difficult or even impossible to quickly organize a fully functional hospital with full diagnostics, operating theatres, capabilities of advanced treatment in various fields of medicine. The concept of creating a temporary hospital is based on the idea of focusing on patients whose condition will not require advanced treatment of coexisting diseases while relieving the burden of hospitals operating on normal principles, whose task is precisely the treatment of patients requiring complex diagnostics and cooperation of physicians specialists in various fields of medicine. The concept of creating temporary hospitals, which is currently being sometimes criticized, has been tested in other countries, and if the number of patients requiring hospitalization due to COVID-19 increases further, these hospitals may prove extremely useful. The usefulness of a temporary hospital should always be assessed in terms of the number of patients with COVID-19 requiring hospitalization. The experience gained during the development of a temporary hospital will allow for better preparation of further such units and more optimal use of medical personnel and equipment.

Conflict of interest statement: Authors don't declare any conflict of interest. 


\section{REFERENCES}

1. Dzieciatkowski T, Szarpak L, Filipiak KJ, et al. COVID-19 challenge for modern medicine. Cardiol J. 2020; 27(2): 175-183, doi: 10.5603/ CJ.a2020.0055, indexed in Pubmed: 32286679.

2. Saban M, Shachar T. The silent effect of COVID-19 on emergency departments: How to avoid complacency? Disaster and Emergency Medicine Journal. 2020, doi: 10.5603/demj.a2020.0035.

3. Chen $Z$, He $S$, Li F, et al. Mobile field hospitals, an effective way of dealing with COVID-19 in China: sharing our experience. Biosci Trends. 2020; 14(3): 212-214, doi: 10.5582/bst.2020.01110, indexed in Pubmed: 32238673.

4. Murray C. Forecasting the impact of the first wave of the COVID-19 pandemic on hospital demand and deaths for the USA and European Economic Area countries. , doi: 10.1101/2020.04.21.20074732.

5. Sacchetto D, Raviolo M, Beltrando C, et al. COVID-19 surge capacity solutions: our experience of converting a concert hall into a temporary hospital for mild and moderate COVID-19 patients. Disaster Med Public Health Prep. 2020 [Epub ahead of print]: 1-10, doi: 10.1017/ dmp.2020.412, indexed in Pubmed: 33100254.

6. Miller NM, Jones I, Russ $S$, et al. A model for rapid emergency department expansion for the COVID-19 pandemic. Am J Emerg Med. 2020; 38(10): 2065-2069, doi: 10.1016/j.ajem.2020.06.026, indexed in Pubmed: 33142176.

7. Wang W, Xin C, Xiong Z, et al. Clinical Characteristics and Outcomes of 421 Patients With Coronavirus Disease 2019 Treated in a Mobile Cabin Hospital. Chest. 2020; 158(3): 939-946, doi: 10.1016/j. chest.2020.05.515, indexed in Pubmed: 32437696.

8. $X u X W, W u X X$, Jiang XG, et al. Clinical findings in a group of patients infected with the 2019 novel coronavirus (SARS-Cov-2) outside of Wuhan, China: retrospective case series. BMJ. 2020; 368: m606, doi: 10.1136/bmj.m606, indexed in Pubmed: 32075786. 